\title{
Mutual-Interaction Potential Fields Model to Simulate Crowd Behavior during Evacuation Processes
}

\author{
Mohamed H. Mabrouk
}

\begin{abstract}
The fact of overpopulation in many countries drags the attention to the need of predicting the human crowd behaviour through the development of reliable models that can be used to plan building evacuation processes in emergency situations. Computer based models that simulate human crowd dynamics prove to offer the optimum solution in such cases. This paper presents a model to simulate the behaviour of human crowd using the artificial potential fields to describe the interaction forces between each crowd member and the environment on one side and amongst the crowd members on the other side by adopting the mutual interactions rules that appear in many biological systems such as bird flocks and fish schools. Rather than simulating the individual based behaviour of each crowd member, a collective behaviour through the interactions amongst the crowd members is simulated adding more realistic flavour to the predicted crowd behaviour which is vital to plan the evacuation processes of buildings especially those with complex structures such as buildings with narrow corridors and bottlenecks. Numerical results that match the real observed behaviour of human crowds in building evacuation processes prove the efficiency and the applicability of the model.
\end{abstract}

Key words: Physical systems, Modeling and simulation, Pedestrian dynamics, Artificial potential fields, Lyapunov stability.

\section{INTRODUCTION}

Evacuation of a crowded building in emergency situations may result in the loss of lives and the injury of many people. Moreover, the complex internal structure of buildings such as narrow corridors and bottlenecks may render difficulty in speedy escape. Aggregation of people in crowds can be highly unsafe in different situations such as fire alarms, which can become disastrous and many people can be badly injured or killed through crowd pushing forces just as they can be hurt by smoke and flames [1,2]. Also, large numbers of people moving in a constrained environment, even if they are in a non emergency situation, can also find themselves in dangerous conditions such as the gathering of millions of Muslims at the Hajj [3, 4].

The crowd dynamics has been studied over the years using many approaches [5-10], some of which depend on recorded observations and empirical data $[6,10]$, while others use the computer based modeling that proves technical to plan evacuation scenarios for buildings especially those of complex structures as the computer model can be used to examine the effects of different incident assumptions and parameters through its ability of multiple-running [6, 11-13]. Computer models are used in planning designated walking routes for large urban festivals [14], interactions between military and civilian groups in wartime [15], estimating time for people to evacuate a high-rise building [16], and many other applications. There are different approaches for modeling, some of them are based on traditional numerical-continuous techniques adapted from physics [17] while others are based on discrete modelling techniques like cellular automata [18].

The majority of the available studies investigate human crowds as collection of isolated individuals that happen to exist close to each other, rather than individuals who have social ties such as friends or family members, each having his own speed and destination point $[5,6,7,11,19,20]$. A number of simulation models have been proposed such as queuing models [21], transition matrix models [22], and stochastic models [23], which are partly related to each other. In addition, there are models for the route choice behaviour of pedestrians [24]. None of these concepts adequately takes into account the collective behaviour effects occurring in pedestrian 
crowds. However, some models has recently present methods to simulate collective behaviour in crowds such as social force models [25, 26], "cellular automata of pedestrian dynamics" [18, 27] and "AI-based models" [28].

The behaviour of a human crowd in constrained environment seems to have certain properties that emerge from the collective behaviour of individuals within the crowd $[25,26]$. Like aggregations of members in many physical systems that have their own emergent dynamics, there is an interest in determining the basis of collective behaviour effects and properties of large groups of people that move through restricted environments such as narrow corridors and bottlenecks. Considering crowd behaviour especially in emergency situations, it is worth noting that the groups of humans are driven by social forces, which are mainly resulted from mutual interactions amongst the crowd members in a way that appears in many other physical systems such as animal herds, fish schools, bird flocks and ant colonies [29].

The model presented here [30] is considered as an extension of earlier work published by the author [5, 7], in which the forces amongst the crowd members were simulated on an individual basis. In this paper, the social potentials model is used to predict the crowd behaviour during challenging evacuation situations. The social forces amongst the crowd individuals are taken into consideration to add extra realistic flavour to the predicted crowd behaviour as will be explained in section II. In scetion III, the stability of the system is discussed. Section IV presents the numerical results and discussion. Finally, conclusions are presented in section V.

\section{THE MUTUAL-INTERACTION POTENTIAL FIELDS MODEL}

As crowd behaviours arise from interactions amongst members of large groups of people, computer modelling has become a key tool to study the foundations of crowd behaviour and to predict crowd movement through different environments. Many of these models are based on the 'physics' of crowd motion [31, 32]. The idea that many physical systems attempt to relax their configuration to a minimum-energy state was adopted in my previous work $[5,7]$ to successfully simulate the crowd behavior using the artificial potential field method for describing the interactions amongst members in crowds on an individual basis.

The artificial potential fields (APF) has been used in models that describe the interactions amongst the members of physical systems that depend on weak long-range attraction and strong short-range repulsion where both attractive and repulsive fields are formed separately and then added to form a global potential field [5, 7, 33]. Genralized Morse potential (GMP) is preferred to be used for both the attractive and repulsive potentials because its exponentially decaying nature which defines a realistic way of constructing the field [33-35] as well as its simpler computations required especially when dealing with a large number of individuals, avoiding disadvantages of former attempts that were presented in the field of pedestrian dynamics which either did not present realistic way to define the interactions amongst the crowd members [36,37] or were based on methods that demand high calculations especially for large crowds [38, 39].

The model in this paper is considered for the $i^{\text {th }}$ individual with mass $m_{i}$, position $\mathbf{r}_{i}$ and velocity $\mathbf{v}_{i}$. The model includes a dissipative force $\left(-\beta_{i} \mathbf{v}_{i}\right)$, which is similar to simple friction that directly acts upon the $i^{\text {th }}$ individual, to prevent group members from violent motion within the group, the positive coefficient $\beta_{i}$ is chosen to control the $i^{\text {th }}$ individual's speed. The potential fields are defined by assigning repulsive potentials (repulsive forces) to the obstacle positions while assigning attractive potentials (attractive forces) to the goal position. The model components which determine behaviour of each individual in each time period of the simulation are defined to achieve collective behaviour amongst the crowd members by assigning social potentials of weak long-range attraction and strong short-range repulsion between each individual and the other crowd members. The environmental potential affecting the $i^{\text {th }}$ individual is characterised by attractive goal and repulsive obstacle potential fields of strengths $C_{i g}$ and $C_{i o}$ with ranges $l_{i g}$ and $l_{i o}$ respectively, where $C_{i g}, C_{i o}, l_{i g}$ and $l_{i o}$ are positive constants. The interaction potential is characterised by constant attractive and repulsive potential fields of strength $C_{a}$ and $C_{r}$ with ranges $l_{a}$ and $l_{r}$ respectively, where $C_{a}, C_{r}, l_{a}$ and $l_{r}$ are positive constants. The ratios $l$ and $C$, which are the potential range ratio and the potential strength ratio, are defined to equal $\left(l_{r} / l_{a}\right)$ and $\left(C_{r} / C_{a}\right)$ respectively. The equations of motion of the $i^{\text {th }}$ individual are as follows: 
$\mathbf{v}_{i}=\dot{\mathbf{r}}_{i}$

$m_{i} \dot{\mathbf{v}}_{i}=-\beta_{i} \mathbf{v}_{i}-\nabla_{i} V_{\text {global }}\left(\mathbf{r}_{i}\right)$

where the global potential function $V_{\text {global }}\left(\mathbf{r}_{i}\right)$ corresponding to the $i^{\text {th }}$ individual can be defined as:

$V_{\text {global }}\left(\mathbf{r}_{i}\right)=\sum_{j \neq i}^{N_{p}} C_{r_{j}} e^{-\left|\mathbf{r}_{i}-\mathbf{r}_{j}\right| / l_{r_{j}}}-\sum_{j \neq i}^{N_{p}} C_{a_{j}} e^{-\left|\mathbf{r}_{i}-\mathbf{r}_{j}\right| / l_{a_{j}}}+\sum_{z=1}^{N_{o}} C_{i o_{z}} e^{-\left|\mathbf{r}_{i}-\mathbf{r}_{o_{z}}\right| / l_{i_{z}}}-C_{i g} e^{-\left|\mathbf{r}_{i}-\mathbf{r}_{g}\right| / l_{i g}}$

where $N_{o}$ is the total number of point obstacles that constitute the boundaries (walls), $N_{p}$ is the number of crowd members, $\mathbf{r}_{g}$ is the goal position and $\mathbf{r}_{o z}$ is the $z^{\text {th }}$ obstacle point position. This way of defining the model elements has the advantages of taking the physical terms of velocity and acceleration into account as well as the relatively simple required calculations. It also gives the model the advantages of adaptation to real situations especially in crisis situation, in addition to the employment of generalized morse potential to define the social forces amongst the individuals that gives more realistic flavour to the model.

Figure1 shows an example of the interaction potential, which represents weak long-range attraction and strong short-range repulsion interactions. The domination of the repulsion potential at low inter-member distances and the domination of the attraction potential at large inter-member distances, which appear in interactions amongst members of many biological systems [40], is clear in figure1. The comfortable distance $d^{*}$, which is the distance between two interacting agents resulting from the equilibrium of the repulsion and attraction interaction potentials terms in Eq. (3), can now be calculated for the model as follows [40]:

$d^{*}=\frac{l_{a} l_{r}}{\left(l_{a}-l_{r}\right)} \ln \left(\frac{C_{r}}{C_{a}}\right)$

The comfortable distance $d^{*}$, which occurs when repulsion and attraction potential equilibrate, is shown in Figure1. An explicit expression for the social interaction forces $\mathbf{F}_{\text {interaction }}\left(\mathbf{r}_{i}\right)$ acting on $i^{\text {th }}$ agent, due to the social potentials is:

$\mathbf{F}_{\text {interaction }}\left(\mathbf{r}_{i}\right)=\sum_{j \neq i}^{N_{p}}\left(\frac{C_{r_{j}}}{l_{r_{j}}} e^{-\left|\mathbf{r}_{i}-\mathbf{r}_{j}\right| / l_{r_{j}}}-\frac{C_{a_{j}}}{l_{a_{j}}} e^{-\left|\mathbf{r}_{i}-\mathbf{r}_{j}\right| / l_{a_{j}}}\right) \hat{\mathbf{r}}_{i j}$

where $\hat{\mathbf{r}}_{i j}$ is the unit vector in the direction of the vector $\mathbf{r}_{i j}$. In an (obstacles/goals) free environment, the total force $\mathbf{F}_{\text {total }}\left(\mathbf{r}_{i}, \mathbf{v}_{i}\right)$ that acts upon the $i^{\text {th }}$ individual amongst the crowd according to the model is defined as:

$\mathbf{F}_{\text {total }}\left(\mathbf{r}_{i}, \mathbf{v}_{i}\right)=-\beta_{i} \mathbf{v}_{i}+\sum_{j \neq i}^{N_{p}}\left(\frac{C_{r_{j}}}{l_{r_{j}}} e^{-\left|\mathbf{r}_{i}-\mathbf{r}_{j}\right| / l_{r_{j}}}-\frac{C_{a_{j}}}{l_{a_{j}}} e^{-\left|\mathbf{r}_{i}-\mathbf{r}_{j}\right| / l_{a_{j}}}\right) \hat{\mathbf{r}}_{i j}$ 


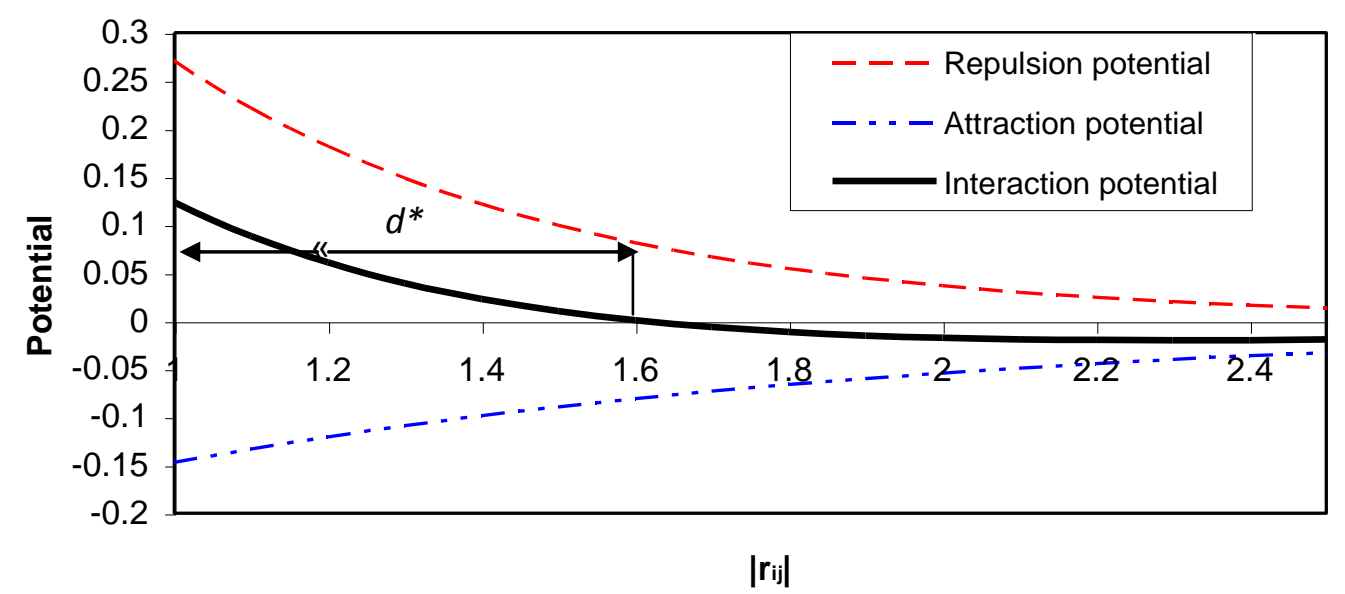

Fig.1 The social interaction potentials for parameters $\left(C_{a}=0.4, C_{r}=2, l_{a}=1, l_{r}=0.5\right)$

STABILITY ANALYSIS

The approach adopted to prove the stability of a system of interacting particles [40] is now used to prove that the behaviour of crowd members' simulated by the social potentials model is Lyapunov stable, where the system members tend to relax into a minimum energy state [41].

Firstly, we will discuss the model during the regime when the only forces that affect the crowd members are the social interaction forces. Considering the potential field defined in Eq. (3), the potential field is now a function of the social potential interaction parameters $C_{a}, C_{r}, l_{a}$ and $l_{r}$. According to the equations of motion and assuming unit mass, the equation of motion of a single individual $i$ at position $\mathbf{r}_{i}$ is:

$$
\ddot{\mathbf{r}}_{i}=-\beta_{i} \mathbf{v}_{i}-\nabla_{i} V_{\text {global }}\left(\mathbf{r}_{i}\right)
$$

where $V_{\text {global }}\left(\mathbf{r}_{i}\right)$ is the global potential that affects the $i^{\text {th }}$ individual. From Eq. (3) and for an (obstacles / goals) free environment, the global potential function for a single individual at position $\mathbf{r}_{i}$, which is affected only by the social forces amongst the crowd, can be defined as:

$$
V_{\text {global }}\left(\mathbf{r}_{i}\right)=\sum_{j \neq i}^{N_{p}} C_{r_{j}} e^{-\left|\mathbf{r}_{i}-\mathbf{r}_{j}\right| / l_{r_{j}}}-\sum_{j \neq i}^{N_{p}} C_{a_{j}} e^{-\left|\mathbf{r}_{i}-\mathbf{r}_{j}\right| / l_{a_{j}}}
$$

The system effective energy $\phi$, for $N_{p}$ members of the crowd, will be defined as follows:

$$
\phi=\sum_{i=1}^{N_{p}}\left(\frac{1}{2} \mathbf{v}_{i}^{2}+V_{\text {global }}\right)
$$

so that its time derivative is 
$\dot{\phi}=\sum_{i=1}^{N_{p}}\left(\mathbf{v}_{i} \cdot \dot{\mathbf{v}}_{i}+\nabla_{i} V_{\text {global }} \cdot \mathbf{v}_{i}\right)$

Substituting from Eq. (2) in Eq. (10), it can be seen that

$$
\dot{\phi}=-\beta \sum_{i=1}^{N_{p}}\left(\mathbf{v}_{i}^{2}\right)
$$

Since $\beta>0$, it is clear that $\dot{\phi}<0$ and therefore the system is Lyapunov stable [40, 41].

Secondly, the tendency of crowd individuals to reach the goal position in an environment, which contains obstacles and a goal point, is discussed on an individual basis to simplify the problem. According to Eq. (1-3) and for a single individual at position $\mathbf{r}_{i}$, it can be seen that:

$$
\ddot{\mathbf{r}}_{i}=-\beta \mathbf{v}_{i}-\nabla_{i} V_{\text {goal }}\left(\mathbf{r}_{i g}\right)-\nabla_{i} V_{\text {obstacles }}\left(\mathbf{r}_{i o}\right)
$$

where $V_{\text {goal }}\left(\mathbf{r}_{i g}\right)$ is the potential field of a single goal and $V_{\text {obstacles }}\left(\mathbf{r}_{i o}\right)$ is the obstacles potential field that affect the $i^{\text {th }}$ individual. However, from Eq. (3) it can be seen that

$$
V_{\text {obstacles }}\left(\mathbf{r}_{i o}\right)=\sum_{z=1}^{N_{o}} C_{i o_{z}} e^{-\left|\mathbf{r}_{i}-\mathbf{r}_{o_{z}}\right| / l_{i_{z}}}
$$

where $N_{o}$ is the number of obstacles. Then, from Eq. (13) it can be seen that

$$
\nabla_{i} V_{\text {obstacles }}\left(\mathbf{r}_{i o}\right)=-\sum_{z=1}^{N_{o}} \frac{C_{i o_{z}}}{l_{i o_{z}}} e^{-\left|\mathbf{r}_{i}-\mathbf{r}_{o_{z}}\right| / l_{i o_{z}}} \hat{\mathbf{r}}_{i o_{z}}
$$

Noting that $C_{i o}$ is given very small values compared to $C_{g}$ to avoid formation of local minimum inside the trap [7], then the term $\nabla_{i} V_{\text {obstacles }}\left(\mathbf{r}_{i o}\right) \approx 0$, then Eq. (12) will approximate as

$$
\ddot{\mathbf{r}}_{i}+\beta \dot{\mathbf{r}}_{i}=-\nabla_{i} V_{\text {goal }}\left(\mathbf{r}_{i g}\right)
$$

which is the equation for a damped oscillator (due to the linear dissipation term) with an external forcing term generated by the goal [42]. This indicates the tendency of each individual to move to the goal position and then come to rest.

\section{NUMERICAL RESULTS AND DISCUSSION}

From the recorded observations of real behaviour of humans [6], the behaviour of people in emergency situations is too unmanageable to be predicted because when people panic, they get nervous and try to escape in a speedy random fashion. However, if people do not panic in emergency situations, they seem to follow the crowd members, who succeed to evacuate, with relatively higher speed than that in the normal conditions [6]. 


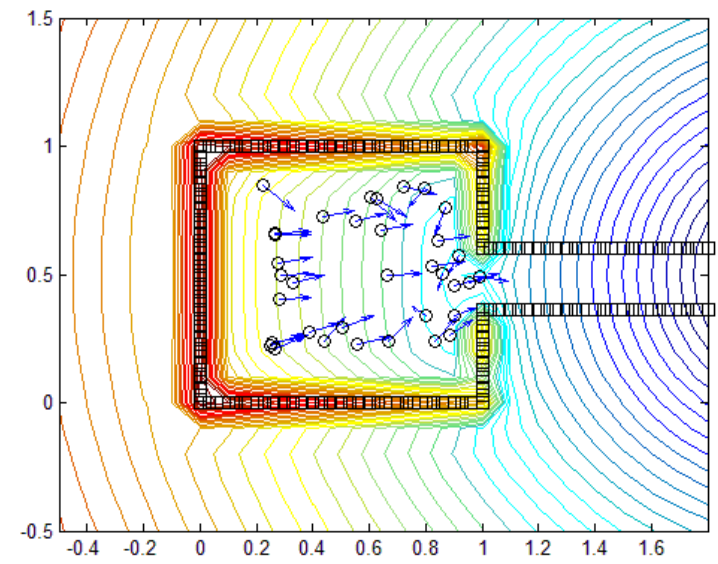

(a) $t=10$

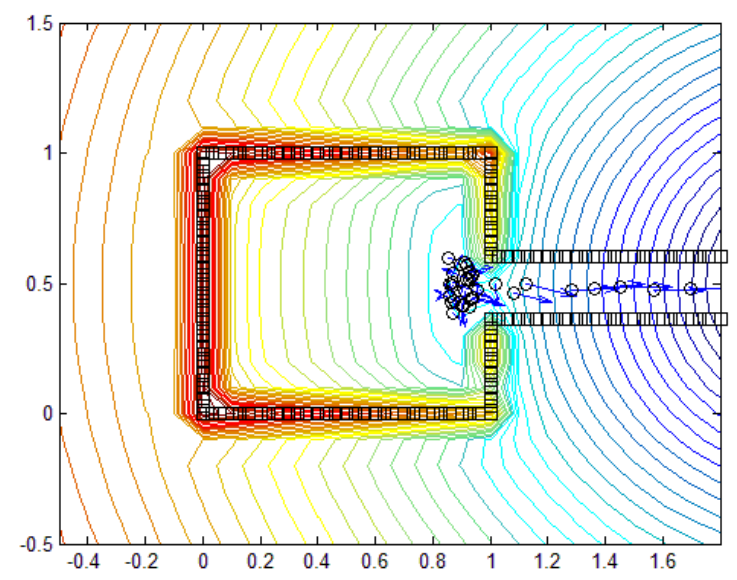

(c) $t=70$

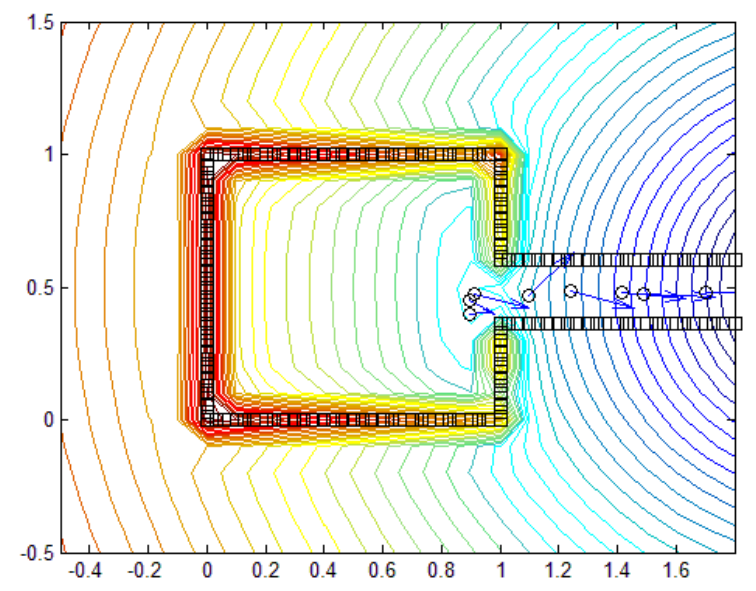

(e) $t=140$

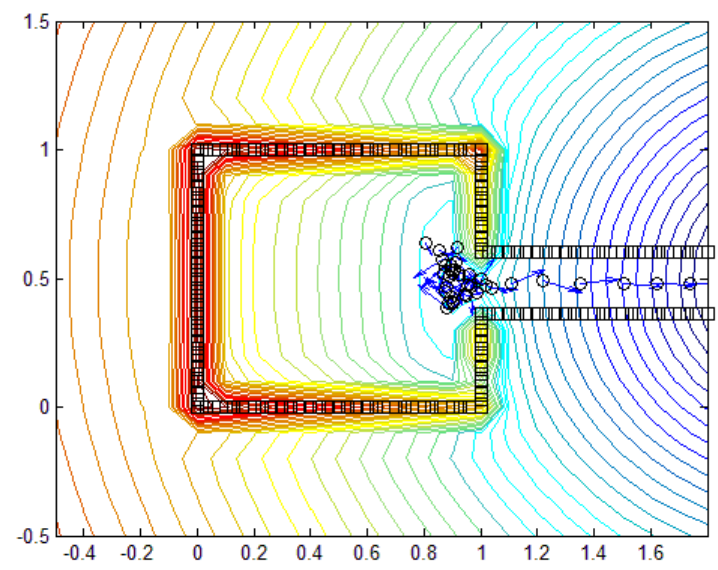

(b) $t=55$

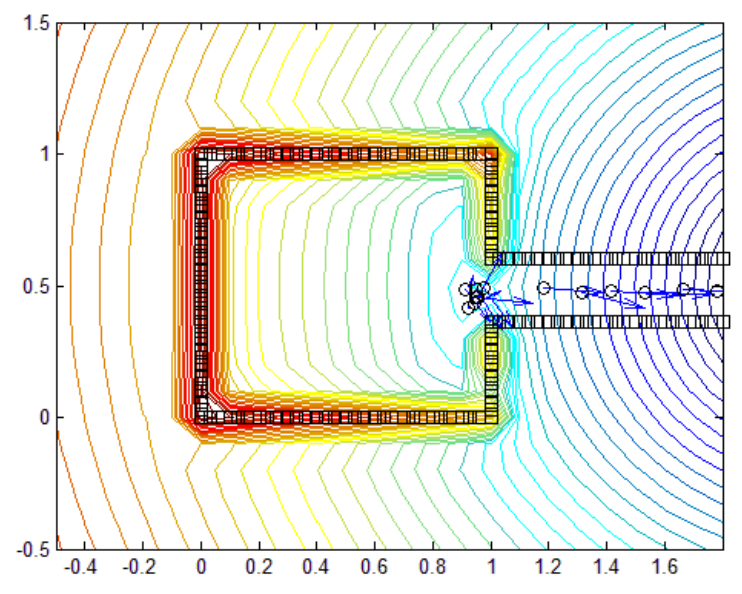

(d) $t=125$

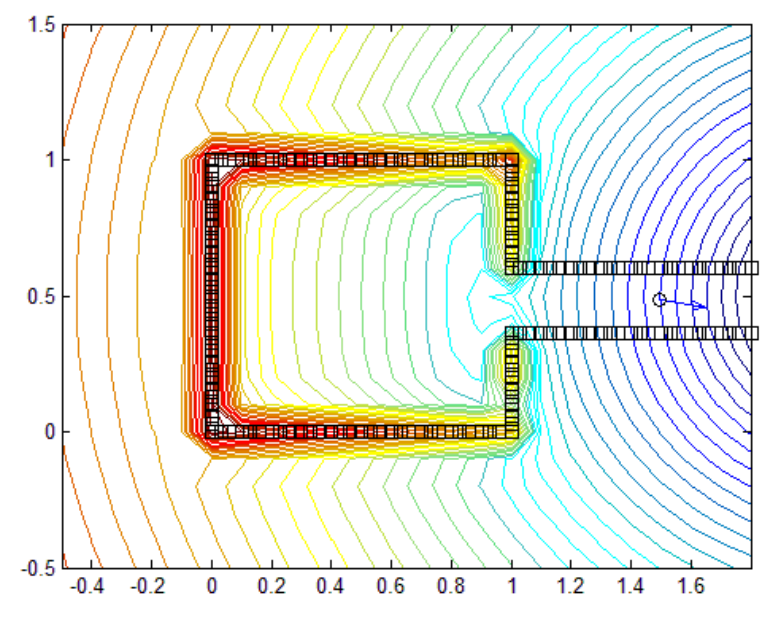

(f) $t=147$

Fig.2. Simulation of crowd members that attempt to leave a trap to the exit and succeed, $t=0: 147$ 
The simulation results shown in Fig. 2 demonstrate the behaviour of a 35 individuals crowd from random initial positions and velocities during an evacuation situation from a room passing through a bottleneck then along narrow corridor that leads to the exit. Buildings with such complex structures are challenging during the planning of evacuation processes according to the reactive problem that may occurs and some of the individuals may be trapped in one of the corners which is considered as a drawback of using artificial potential field method in such cases. This problem is taken into consideration when choosing the social potential interaction parameters of the global potential field function. The assigning of faint repulsion potential from the walls while assigning strong goal attraction potential proves effective to solve the problem [7] and lead all the crowd members to the exit as shown in Fig. 2.

From the simulation results shown in Fig. 2, it can be seen that some individuals pass the bottleneck according to their close initial positions to the neck as shown in Fig. 2(a), hence the rest of the individuals seem to follow them and aggregate around the neck as shown in Fig. 2(b-d). Following the succeeding individuals is due to the weak long-range attraction social potential amongst the crowd members which helps the aggregated members at the bottleneck to evacuate the room and to pass the corridor in a line which can be clearly seen in Fig. 2(b-e). The process continues until each individual reaches the destination point while keeping a distance from its nearest neighbour in the crowd, as shown in Fig. 2, due to the strong short-range repulsion social potential amongst the crowd members. It is worth noting that as the evacuation process continues, the number of individuals left in the room decreased which leaves larger spaces amongst the individuals and the evacuation process becomes faster with time as can be noted in Fig. 2 (d-f).

Crowd members then tend to come to rest when reaching the goal point in a way that matches the stability analysis of the model which proves that the crowd members tend to relax into a minimum energy state at the goal position. This achieves the real features of crowd members, who desire not to move too far and tend to reach their destinations through the shortest path while avoiding other members in the crowd. Also this matches the observed data that attempted to be simulated by other models [43-45] yet with lower calculations required.

Figure 3 demonstrates the behaviour of human crowd members that attempt to leave a room through a narrow corridor from random initial positions and velocities. Figure 3.a) shows the crowd predicted behaviour simulated by the model, while Fig.3.b) shows a formerly published [46] recorded observation of real human crowd in same situation. The simulated behaviour of the crowd predicted by the model seem to be similar to recorded observations for real behaviour of human crowd as can be seen in individuals' behaviour of their aggregation around the neck, keeping a distance from each others, and leaving the room in a line.

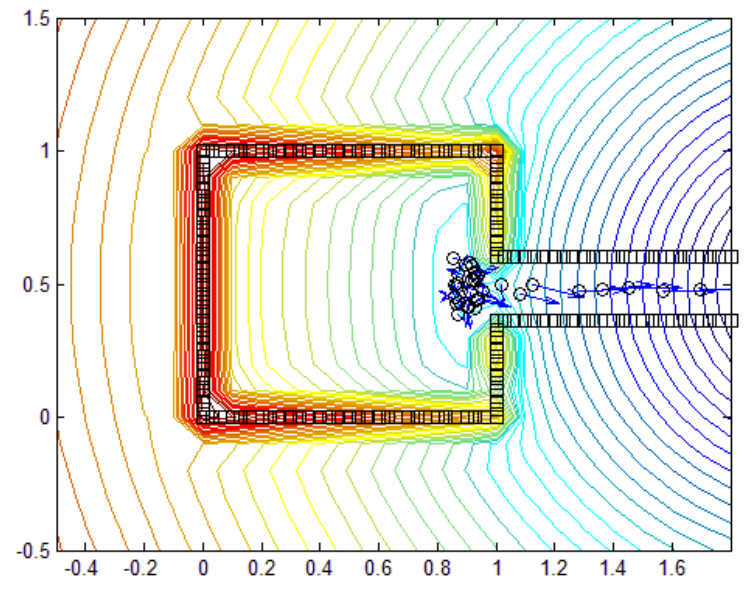

(a)

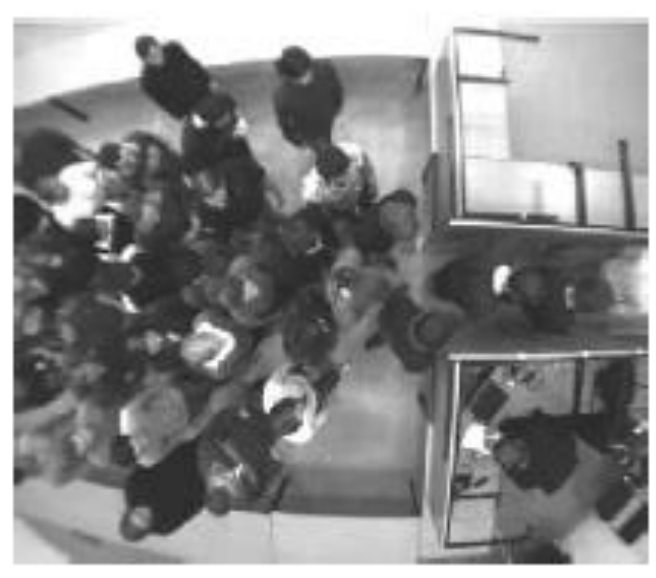

(b)

Fig.3 Simulation of crowd behaviour at bottleneck using the social potentials model versus video recordings [46] of real crowd behaviour in similar situation. 


\section{CONCLUSIONS}

This paper presents a computer based model to predict human crowd dynamics during evacuation processes. The model is considered as a modification of previous work, which predict crowd behaviour on an individual basis [5, 7], through the inclusion of the social interaction forces amongst the crowd members. The model employs the weak long-range attraction potentials and strong short-range repulsion potentials to express the social forces amongst the crowd members mimicking the interaction forces that appear in many biological systems such as animal herds and ant colonies. The model uses an efficient way to describe the motion through taking the physical terms of velocity and acceleration of each crowd member into account. Also, the possibility to alter the parameters in the global potential equation, which represents the environment in which the crowd exist, makes the model capable of avoiding the reactive problem that is considered as a common drawback when simulating the human crowd behaviour in buildings with complex structures such as buildings with narrow corridors and bottlenecks. This way of defining the model elements gives the model the advantages of adaptation to real human behaviour as well as the relatively simple required calculations. The stability analysis proves that, using the social potentials model, crowd members tend to relax into a minimum energy state which achieves the real behaviour of human pedestrians who desire to reach their destinations through the shortest path while avoiding the obstacles and other individuals. The numerical results which match the real observed data in similar situation show the ability of the model to predict the human crowd behaviour in a way that it can be used effectively to plan the evacuation processes of complex structured buildings with the possibility of changing many parameters to adapt the different emergency situations scenarios.

\section{REFERENCES}

[1] Chertkoff, J., and Kushigian, R., "Don’t panic: The psychology of emergency egress and ingress". London: Praeger, (2001).

[2] BBC. News, "Blaze at Qatar shopping centre kills 19", http://m.bbc.co.uk/news/world-middle-east18243136?SThisFB, (2012).

[3] Crowd Dynamics. Crowd disasters. Retrieved 2008-08-10 from http://www.crowddynamics.com/Main/Crowddisasters.html, (2007).

[4] Anders Johansson, Michael Batty, Konrad Hayashi, Osama Al Bar, David Marcozzi and Ziad A Memish, "Crowd and environmental management during mass gatherings", www.thelancet.com/infection, Online Publications. DOI:10.1016/S1473-3099(11)70287-0, (2012).

[5] Mohamed H. Mabrouk, "Individual-based model to simulate crowd dynamics using artificial potential fields," Proc. of the World Congress on Engineering 2011 (WCE2011), Vol III, pp 1973-1980 (2011).

[6] Helbing, D., “ Traffic and related self-driven many-particle systems,” Reviews of Modern Physics,V.73, (2001)

[7] Mohamed H. Mabrouk, "Using Successive Multi-Goals Method in the Individual-Based Model to Simulate Crowd Behavior during Evacuation Processes", Journal of Mechanics Engineering and Automation, V.1, No.4, 325-330, (2011).

[8] Hoogendoorn, S. and Daamen, W., "Pedestrian behaviour at bottlenecks," Transportation Science, 39: 147159, (2005).

[9] Kretz, T., "Pedestrian traffic: On the quickest path," Journal of Statistical Mechanics: Theory and Experiment, P03012, (2009).

[10] Moussaid, M., Helbing, D., Garnier, S., Johansson, A., Combe, M., et al., "Experimental study of the behavioural mechanisms underlying self-organization in human crowds," Proc. of the Royal Society B: Biological Sciences, 276: 2755-2762, (2009).

[11] Antonini, G., Bierlaire, M., and Weber, M., "Discrete choice models of pedestrian walking behaviour," Transportation Research Part B 40: 667-687, (2006). 
[12] Castle, C., "Guidelines for assessing pedestrian evacuation software applications," Ucl working papers series, paper 115, ISSN 1467-1298, (2007).

[13] Chraibi, M. and Seyfried, A., "Pedestrian dynamics with event-driven simulation," Pedestrain and evacuation dynamics, Part 2, 713-718, DOI: 10.1007/978-3-642-04504-2_69, (2010).

[14] Batty, M., Desyllas, J., and Duxbury, E, "Safety in numbers? modelling crowds and designing control for the Notting Hill carnival”, Urban Studies, 40, 1573-1590, (2003).

[15] Nguyen, Q.-A. H., McKenzie, F. D., and Petty, M. D., "Crowd behaviour cognitive model architecture design", In Proceedings of the 14th conference on behaviour representation in modeling and simulation (BRIMS-05),(2005).

[16] Fahy, R., "EXIT89 - an evacuation model for high-rise buildings: Model description and example applications", In T. Kashiwagi (Ed.), Proceedings of the 4th international symposium: international association for fire safety science (p. 657-668), (1994).

[17] Helbing, D., Farkas, I. J., Molnár, P., and Vicsek, T., "Simulation of pedestrian crowds in normal and evacuation situations", In M. Schreckenberg \& S. Sharma (Eds.), Pedestrian and evacuation dynamics (pp. 21-58). New York: Springer, (2002).

[18] Burstedde, C., Klauck, K., Schadschneider, A., and Zittartz, J., "Simulation of pedestrian dynamics using a two-dimensional cellular automaton", Physica A, 295, 507-525, (2001).

[19] Seyfried, A., Boltes, M., Kahle, J., Klingsch, W., Portz, A., Rupprecht, T., Schadschneider, A., Steffen, B., and Winkens, A., "Enhanced empirical data for the fundamental diagram and the flow through bottlenecks," Pedestrian and Evacuation Dynamics, Springer, (2008).

[20] Schultz, M., Kretz, T., and Fricke, H., "Solving the direction field for discrete agent motion," in Bandini, S., et al. [Cellular Automata for Research and Industry], (ACRI) 2010, Springer Berlin / Heidelberg, (2010).

[21] Yuhaski SJ Jr, Macgregor Smith JM, “ Modelling circulation systems in buildings using state dependent queueing models", Queueing Syst 4:319-338,(1989)

[22] Garbrecht D., "Describing pedestrian and car trips by transition matrices", Traffic Q 27:89-109

[23] Ashford N, O'Leary M, McGinity PD, "Stochastic modelling of passenger and baggage flows through an airport terminal”, Traffic Engin Control 17:207-210, (1973)

[24] Borgers A, Timmermans H, "City centre entry points, store location patterns and pedestrian route choice behaviour: A microlevel simulation model", Socio-Econ Plan Sci 20:25-31, (1986).

[25] Helbing D, Molnár P, "Social force model for pedestrian dynamics", Phys Rev E 51:4282-4286, (1995)

[26] Helbing D, Molnár P, Farkas I, Bolay K, "Self-organizing pedestrian movement", Env Planning B 28:361383, (2001)

[27] FukuiM, Ishibashi Y, "Self-organized phase transitions in cellular automaton models for pedestrians", J Phys Soc Japan 68:2861-2863, (1999).

[28] Reynolds CW, "Evolution of corridor following behaviour in a noisy world", In: Cliff D, Husbands P, Meyer J-A, Wilson S (eds) From animals to animats 3: Proceedings of the third international conference on simulation of adaptive behavior. MIT Press, Cambridge, pp 402-410, (1994).

[29] Couzin I. and Franks N, "Self-organised lane formation and optimized traffic flow in army ants" In Proc. of the Royal Society of London B 270, 139-146, (2003).

[30] Mohamed H. Mabrouk, "Social Potential Fields Model to Simulate Pedestrian Dynamics", Submitted to Pedestrain and evacuation dynamics, (2012).

[31] Helbing, D., Johansson, A., and Al-Abideen, H., "The dynamics of crowd disasters: an empirical study," Physical Review E, 75: 046109, (2007).

[32] Yu, W. and Johansson, A., "Modeling crowd turbulence by many-particle simulations," Physical Review E, 76: 046105, (2007)

[33] Mohamed H. Mabrouk, and Colin R. McInnes, "Non-linear stability of vortex formation in swarms of interacting particles," Physical Review E, 78: 012903, (2008).

[34] D’Orsogna, M. R., Chuang, Y. L., Bertozzi, A. L., and Chayes, L., "Self-propelled agents with soft-core interactions: patterns, stability, and collapse," Phys. Rev. Lett., vol. 96, 104302, (2006).

[35] McInnes, C., "Vortex formation in swarm of interacting particles," Physical Review E, 75: 032904, (2007). 
[36] Schadschneider, A., "Bionics-inspired cellular automaton model for pedestrian dynamics," in Fukui, M., Sugiyama, Y., Schreckenberg, M., and Wolf, D., eds., [Traffic and Granular Flow], Springer-Verlag Berlin Heidelberg, Nagoya, pp. 499-509, (2003).

[37] Nishinari, K., Kirchner, A., Namazi, A., and Schadschneider, A., "Extended floor field ca model for evacuation dynamics," IEICE Trans. Inf. \& Syst., E87-D, 726-732, cond-mat/0306262v1, (2004).

[38] Treuille, A., Cooper, S., and Popovic, Z., "Continuum crowds," Proc. of the Association for Computing Machinery's Special Interest Group on Graphics and Interactive Techniques , pp 1160-1168, (2006).

[39] Kretz, T., Bönisch, C., and Vortisch, P., "Comparison of various methods for the calculation of the distance potential field," in [Pedestrian and Evacuation Dynamics] 2008, Klingsch, W.W.F., Rogsch, C., Schadschneider, A., and Schreckenberg, M., eds., pp 335-346. Springer-Verlag Berlin Heidelberg. ISBN: 978-3-642-04503-5, (2010).

[40] Mogilner A., Edelstein-Keshet L., Bent L. and Spiros, A., "Mutual interactions, potentials and individual distance in a social aggregation", Journal of Theoretical Biology 47, 353-389, (2003).

[41] Hale, J., "Asymptotic behaviour of dissipative systems", American Mathematical Society, Providence, Rhode Island, (1988).

[42] P. Beer, E. Johnston, and W. Clausen, "Vectors mechanics for engineers: Dynamics," McGraw-Hill Higher Education. ISBN 978-007-127360-2, (2007).

[43] M. Batty,“Agent-based pedestrian modelling”,Ucl working papers series, p. 61, ISSN 1467-1298, (2003).

[44] P. Scovanner and M. Tappen, "Learning pedestrian dynamics from the real world," IEEE International Conference on Computer Vision, ISSN: 1550-5499, 381-388, (2009)

[45] T. Kretz, "The Dynamic distance potential field in a situation with asymmetric bottleneck capacities," in S. Bandini et al. Cellular Automata for Research and Industry (ACRI) 2010, Springer Berlin / Heidelberg, (2010).

[46] Schadschneider, A., et al., "Evacuation dynamics: empirical results, modeling and applications," in Encyclopedia of Complexity and System Science, arXiv:0802.1620v1, (2008) 